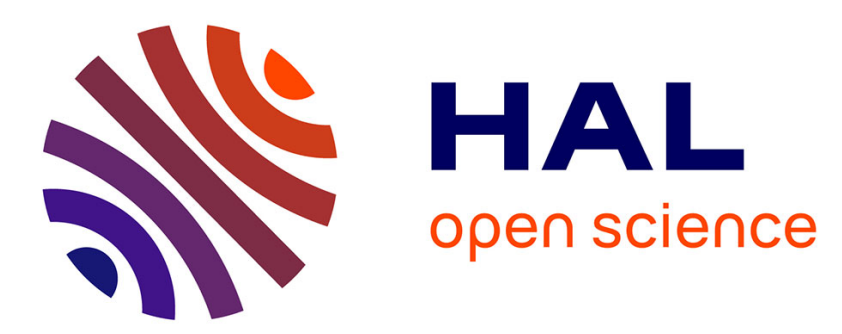

\title{
Semper en version française: traductions et interprétations dans la seconde moitié du XIXe siècle
}

Estelle Thibault

\section{To cite this version:}

Estelle Thibault. Semper en version française: traductions et interprétations dans la seconde moitié du XIXe siècle. Revue Germanique Internationale, 2017, Gottfried Semper. Architecture et anthropologie au XIXe siècle, 26, pp.187-206. halshs-01693046

\section{HAL Id: halshs-01693046 \\ https://shs.hal.science/halshs-01693046}

Submitted on 25 Jan 2018

HAL is a multi-disciplinary open access archive for the deposit and dissemination of scientific research documents, whether they are published or not. The documents may come from teaching and research institutions in France or abroad, or from public or private research centers.
L'archive ouverte pluridisciplinaire HAL, est destinée au dépôt et à la diffusion de documents scientifiques de niveau recherche, publiés ou non, émanant des établissements d'enseignement et de recherche français ou étrangers, des laboratoires publics ou privés. 


\section{Semper en version française : traductions et interprétations dans la seconde moitié du $\mathrm{XIX}^{\mathrm{e}}$ siècle}

Estelle Thibault

Gottfried Semper. Architecture et anthropologie au XIXe siècle. Revue germanique internationale, $\mathrm{n}^{\circ} 26$, Paris, CNRS éditions, 2017, p. 187-206.

p. 187 Comment s'est opérée la diffusion des théories de Gottfried Semper dans la France du XIX ${ }^{\mathrm{e}}$ siècle ? Étant donné que les traductions et recherches sur Semper en anglais ont, ces dernières décennies, précédé celles effectuées en langue française, la réception de ses idées hors du monde germanophone est aujourd'hui mieux connue pour ce qui concerne le milieu architectural anglo-américain ${ }^{1}$. Le rôle qu'a joué l'environnement artistique et scientifique français dans la formation intellectuelle de Semper ${ }^{2}$ invite pourtant à reconsidérer l'importation ultérieure de ses idées vers l'espace culturel francophone. Le fait que l'architecte allemand et ses homologues français partagent un certain nombre de références peut en effet apparaître comme une condition favorable à la discussion de ses idées. L'aspect le mieux cerné concerne la réception française de l'essai de 1834 sur la polychromie, objet d'un compte rendu très critique par Désiré Raoul-Rochette ${ }^{3}$. Mais bien que les premiers écrits de Semper soient connus d'archéologues philologues dont la culture est multilingue, le débat parisien sur la coloration des temples antiques, tel qu'il se diffuse plus largement notamment chez les architectes -, se focalise sur des travaux en français plus aisément accessibles, essentiellement ceux de Jacques Ignace Hittorff. Ainsi, si les échanges européens sur l'historiographie de l'Antiquité ainsi que la question des arts industriels au lendemain de l'exposition universelle de Londres de 1851 prédisposent le public à la compréhension des recherches de Semper, l'existence de travaux voisins en français pourraient en avoir relativisé la singularité.

Nous nous intéresserons ici aux vecteurs de la médiation des ouvrages de Semper à partir d'un examen des publications de la seconde moitié du XIX $^{\mathrm{e}}$ siècle. L'investigation s'est déployée à partir des rares traductions connues, mais dont le contexte d'élaboration méritait d'être précisé, notamment pour la première véritable version française d'un essai de Semper, parue en $1865^{4}$. La découverte récente d'écrits traduits dans une revue d'art décoratif des

\footnotetext{
${ }^{1}$ James Duncan Berry, The Legacy of Gottfried Semper : Studies in Späthistorismus, Ph. D., Brown University, 1989.

${ }^{2}$ Voir Wolfgang Herrmann, Gottfried Semper im Exil, Bâle/Stuttgart, Birkhäuser, 1978, p. 10-31 ; Harry F. Mallgrave, Gottfried Semper Architect of the Nineteenth Century, New Haven/Londres, Yale University Press, 1996, pp. 165-182 ; Pierre Bourlier, "Gottfried Semper à Paris », in : Jean-Louis Cohen (éd.), Interférences/ Interferenzen. Architecture Allemagne/ France 1800-2000, Strasbourg, Musées de Strasbourg, 2013, pp. 150156.

${ }^{3}$ Désiré Raoul-Rochette, «Bemerkungen über vielfarbige Architektur und Sculptur bei den Alten, von G. Semper, Altona, $1834 »$, Journal des savants, $1^{\mathrm{er}}$ nov. 1836, pp. 667-684. Voir aussi par exemple Ernest Beulé, «L'architecture au siècle de Pisistrate», Revue générale de l'architecture et des travaux publics (ci-après RGATP), vol. XVI, 1858, (col. 193-212), col.202. Sur ces débats voir notamment David Van Zanten, «Architectural Polychromy : Life in Architecture », in : Robin Middleton (éd.), The Beaux-Arts and Nineteenth Century Architecture, Cambridge Mass., MIT Press, 1982, pp. 196-215 ; Marie-Françoise Billot, « Recherches aux XVIII ${ }^{\mathrm{e}}$ et $\mathrm{XIX}^{\mathrm{e}}$ siècles sur la polychromie de l'architecture grecque, in : Paris Rome Athènes. Le voyage en Grèce des architectes français aux XIX $X^{e}$ et $X X^{e}$ siècles, Paris, ENSBA, 1982, pp. 61-125.

${ }^{4}$ "Esthétique. Cours de M. Semper: de l'ornementation et du style, de leur signification symbolique dans l'art », Revue des cours littéraires de la France et de l'Étranger, t. $11, \mathrm{n}^{\circ} 32$ et 33, 8 et 15 juil. 1865, p. 513-520 et 539-442 (traduction, par Paul Challemel-Lacour, de « Über die formelle Gesetzmässigkeit des Schmuckes und
} 
années $1880^{5}$ nous a conduit à enquêter plus systématiquement dans divers périodiques et ouvrages. Aussi a-t-il été possible de repérer, autour de ces traductions proprement dites, un ensemble de comptes rendus, extraits, paraphrases et commentaires. Les multiples adaptations de Semper qui circulent, plus ou moins fidèles à la version originale, transforment le contenu textuel et iconographique, comme c'est le cas pour bien d'autres « traductions $»^{6}$ dans le domaine de l'architecture. Dans le cas de Semper, l'objectif énoncé de simplifier une langue particulièrement difficile pour la rendre accessible aux lecteurs se trouve bien souvent dépassé par les appropriations inventives des nouveaux auteurs.

En identifiant les acteurs et les destinataires de ces versions, en portant attention aux processus de translation ainsi qu'aux débats dans lesquels ils s'insèrent, nous tenterons de mieux comprendre comment s'interprète en français la pensée de l'architecte allemand. Postérieurement au débat sur la polychromie, deux épisodes de la réception de Semper peuvent être caractérisés. Le premier s'amorce avec son arrivée à Zurich et concerne l'entourage de Marie d'Agoult. Dans le milieu intellectuel républicain du Second Empire se fabrique un premier horizon d'attente de Der Stil, avant même que l'ouvrage ne soit achevé, favorisant l'assimilation de ses idées par la critique artistique. Une seconde séquence s'ouvre autour de 1885, après la mort de Semper, dans le milieu de l'architecture et des arts industriels, avec le concours actif de l'un de ses anciens élèves résidant à Londres, Lawrence Harvey, et de Hans Semper - le fils de l'architecte -, historien de l'art à Innsbruck. Ainsi déplacées dans l'espace culturel français en passant par la Suisse, l'Angleterre et l'Autriche, ses idées se réécrivent et s'adaptent au contexte politique, artistique et architectural.

\section{Marie d'Agoult et Claire de Charnacé : esthétique et critique d'art}

Les amis parisiens de Semper, tels le décorateur Jules Diéterle, ne semblent pas avoir pris part à la diffusion de ses travaux. Autour de 1860, ceux-ci sont surtout discutés dans un milieu littéraire républicain et germanophile, autour de la comtesse Marie d'Agoult et de sa fille, Claire de Charnacé. Elles se sont intéressées à Semper à partir de 18567, au moment où celuici, nommé enseignant à Zurich, fréquente un petit cercle d'intellectuels exilés comme lui, parmi lesquels figurent des proches de Marie d'Agoult, notamment sa fille Cosima, épouse de Richard Wagner, ainsi que le poète Georg Herwegh. Initialement centrée sur la carrière

dessen Bedeutung als Kunstsymbol », Monatsschrift des Wissenschaftlichen Vereins in Zürich, 1856, pp. 101$130)$.

${ }^{5}$ Gottfried Semper, « Science, industrie et art », L'Art pour tous, Bulletin, n 2 à 14, fév. 1886 à fév. 1887, n. p. ; du même, «L'architecture polychrome chez les peuples de l'Antiquité » et «Les quatre éléments de 1'architecture. Essai d'architecture comparée », L'Art pour tous, Bulletin, n 36 à 42, déc. 1888 à juin. 1889, n. p. (traductions, par Émile Reiber, de Wissenschaft, Industrie und Kunst. Vorschläge zur Anregung nationalen Kunstgefühles, Braunschweig, Vieweg und Sohn, 1852 et de Die Vier Elemente der Baukunst. Ein Beitrag zur vergleichenden Baukunde, Braunschweig, Vieweg und Sohn, 1851). Voir Estelle Thibault, «La Science du style face au marché du monde. Les leçons de l'Exposition universelle de Londres, 1851 », in : Gottfried Semper, Science, industrie et art, Gollion, Infolio, 2012, (pp. 7-51), pp. 39-49.

${ }^{6}$ Voir Valérie Nègre, «Le parti de la trahison?», in : Robert Carvais et al. (éd.), Traduire l'architecture : texte et image, un passage vers la création ?, Paris, Picard, 2015, pp. 9-15.

${ }^{7}$ La première évocation de Semper relevée dans la correspondance de Marie d'Agoult avec Herwegh date d'octobre 1856. Voir Marcel Herwegh, Au printemps des dieux. Correspondance inédite de la comtesse Marie d'Agoult et du poète Georges Herwegh, Paris, Gallimard, 1929, p. 163. 
architecturale dresdoise de Semper injustement brisée par l'exil, leur attention se déplace progressivement vers les travaux théoriques.

Dès 1856, Herwegh communique à Claire de Charnacé des renseignements biographiques sur Semper $^{8}$ qui sont utilisés lorsqu'elle publie dans La Presse, sous le pseudonyme de C. de Sault, une série d'articles sur l'actualité artistique en Allemagne'. Le nom de 1'architecte est associé à Dresde, privée d'un talent de premier plan, comparable à celui d'Henri Labrouste et de Félix Duban pour Paris. Elle déplore son exil dans une petite ville comme Zurich: «Qu'est-ce pour un architecte comme Semper d'habiter le pays des chalets?» ${ }^{10}$. Marie d'Agoult s'enquiert régulièrement de ses activités auprès des Herwegh et, suite à l'article paru dans La Presse, il fait parvenir à Claire une « brochure ${ }^{11}$. La comtesse rencontre l'architecte lors d'un séjour en Suisse l'été 1858 :

Semper est un artiste de grand mérite ; il fait de très belles démonstrations, la canne à la main, sur le sable du jardin, et ses opinions que l'on trouve paradoxales sont trop d'accord avec mes instincts pour que je ne trouve pas un plaisir extrême à les lui entendre énoncer dans un discours un peu lourd. Son ouvrage sur les origines de l'architecture, en voie de publication, apprendra beaucoup de choses à beaucoup d'architectes ${ }^{12}$.

En 1859, Claire prépare pour la toute récente Revue germanique un article plus développé sur l'art en Allemagne où « Godefroy Semper ${ }^{13}$ reste lié à Dresde. Elle y évoque le déplacement de son activité vers l'écriture :

Le praticien manque d'occasion de se produire, mais le théoricien n'est pas condamné au silence. Nommé professeur à l'École polytechnique de Zurich, M. Semper ne se laisse point absorber par la science. En dehors de son enseignement, il a recueilli sa pensée exprimée dans divers écrits, et la concentre en un corps de doctrines qui paraîtra prochainement sous ce titre: Théorie et histoire du style dans l'architecture ${ }^{14}$.

Selon Marie d'Agoult, Semper aurait transmis à Claire de nouveaux éléments relatifs à son projet de livre ${ }^{15}$, dont elle espère faire, une fois celui-ci achevé, une présentation étendue :

\footnotetext{
${ }^{8}$ Victor Fleury, Le poète Georges Herwegh (1817-1875), Thèse de doctorat, Université de Paris, 1911, pp. 161162. Herwegh est le « célèbre penseur et connaisseur » cité dans les prolégomènes de Der Stil (Bd. 1, p. XIX) et Victor Fleury traduit dans sa thèse un long extrait inspiré des idées du poète (pp. 322-324).

${ }^{9}$ C. de Sault, « Lettre à un critique. III. Dresde. L'architecte Semper. Le statuaire Rietschel », La Presse, 22 oct. 1856 , n. p.

${ }^{10}$ Ibid.

${ }^{11}$ Marie d'Agoult à Emma Herwegh, 11 janv. 1857 ; Marcel Herwegh, op. cit., p. 174.

${ }^{12}$ Marie d'Agoult à sa fille Blandine, $1^{\text {er }}$ juil. 1858, cité par Jacques Vier, La Comtesse d'Agoult et son temps t. 3, Paris, Armand Colin, 1961, p. 297.

${ }^{13}$ C. de Sault, «De l'art allemand au XIX ${ }^{\mathrm{e}}$ siècle» (2 ${ }^{\mathrm{e}}$ partie), Revue germanique vol. 6, 1959, (p. 613-630), pp. 623-630.

${ }^{4}$ Ibid., p. 629. Ce titre correspond à celui donné à une version de son projet d'ouvrage envoyé à l'éditeur Friedrich Suchland le 29 octobre 1856. Voir Wolfgang Herrmann, op. cit., p. 105.

15 «Nous sommes charmés de l'ouvrage de Semper, mais tâchez de presser les envois et de faire compléter le plus possible les renseignements biographiques. Claire est libre de faire un article aussi étendu que la matière le
} 
« elle saisira d'ici-là toutes les occasions de le citer comme un artiste éminent, et de préparer ainsi le public français à bien accueillir ses idées $\|^{16}$. Aucun compte rendu de Der Stil n'a pu être retrouvé, mais plusieurs études d'esthétique et de critique d'art signées C. de Sault relaient effectivement les théories de Semper. L'un, en 1862, traite "du rôle de l'anthropomorphisme dans l'art religieux $\rangle^{17}$ et s'ouvre en se référant à Der Stil :

De la hache du silex à la bêche du laboureur, de l'amulette du fétichiste à l'Athénè Parthénos, toute création plastique ou poétique du génie de l'homme est œuvre d'art. Mais cette création répond à des besoins d'ordre et d'élévation si divers, qu'on refuse en général le nom d'œuvre d'art aux objets d'un emploi vulgaire, aux essais primitifs de l'industrie naissante et aux premiers signes et symboles matériels du sentiment religieux. Et pourtant ces objets, ces signes et ces symboles sont aux yeux du critique et de l'historien, de véritables productions artistiques. M. G. Semper l'a parfaitement démontré dans son Esthétique pratique ${ }^{18}$.

L'auteur affirme également emprunter à « l'éminent architecte allemand devenu esthéticien » sa méthode pour « arriver à une classification naturelle des productions techniques » :

Étudier ces productions, premièrement comme répondant à un besoin et à un usage déterminé, secondement comme un résultat de la matière première et des procédés de travail. [...] Une série graduée de créations esthétiques correspondant à une série de besoins individuels ou sociaux, tel est donc le tableau général que présente l'art dans l'ensemble de ses manifestations ${ }^{19}$.

Un autre article, consacré en 1865 à une exposition de l'Union des beaux-arts appliqués à l'industrie ${ }^{20}$, formule des analyses sur l'art du vêtement dérivées de Der Stil, sans néanmoins le citer :

Cet art convient en outre à un degré plus haut de sociabilité, car les tissus, d'abord destinés à la personne, forment les premiers meubles de la maison, et quelquefois la maison même: la tente n'étant qu'une construction de tissus attachés tendus et suspendus à des piques, à des lances et à des pieux ${ }^{21}$.

\footnotetext{
comportera, et il me semble qu'il ne fait rien négliger pour la rendre complète ». Marie d'Agoult à Emma Herwegh, [avril] 1858, Marcel Herwegh, op. cit., p. 192.

${ }^{16}$ Marie d'Agoult à Emma Herwegh, 27 mai 1858, Marcel Herwegh, op. cit., p. 195.

${ }^{17}$ C. de Sault, «Du rôle de l'anthropomorphisme dans l'art religieux », Revue germanique, t. 20, 1862, pp. 516538.

${ }^{18}$ Ibid., p. 517. L'article cite, en note, le premier volume de Der Stil ainsi que l'essai de 1856 Über die formelle Gesetzmässigkeit des Schmuckes und dessen Bedeutung als Kunstsymbol [Des lois formelles de la parure et de sa signification comme symbole artistique] (une traduction française de ce texte figure dans G. Semper, Du Style et de l'architecture. Ecrits 1834-1869, trad., choix et introductions de J. Soulillou et N. Neumann, Marseille, Parenthèses, 2007, pp. 235-264).

${ }^{19}$ Ibid.

${ }^{20}$ C. de Sault, « Le musée rétrospectif des arts industriels », Revue moderne, 1865, t. 35, pp. 545-558.

${ }^{21}$ Ibid., p. 551.
} 
La sympathie politique pour l'architecte en exil se double ici d'un intérêt partagé pour des industries ornementales dont l'étude est chargée de significations sociales. Il s'agit de reconsidérer la valeur esthétique de ces productions techniques, à l'égal des œuvres du grand art, et d'inviter à l'union entre le monde des métiers et celui de l'architecture. Cette phase de réception, dans les milieux républicains de la critique d'art des années 1860 et 1870, se focalise ainsi sur la portée symbolique de l'ornement.

\section{De Paul Challemel-Lacour à Charles Blanc: lois et signification symbolique de la parure}

Participant de ces initiatives conjointes pour faire connaître les idées de Semper, la première véritable traduction, publiée en 1865 dans la Revue des cours littéraires, concerne la conférence délivrée à Zurich en janvier 1856, Über die formelle Gesetzmässigkeit des Schmuckes und dessen Bedeutung als Kunstsymbol [Des lois formelles de la parure et de sa signification comme symbole artistique $]^{22}$.

Le traducteur, Paul Challemel-Lacour, fait partie du réseau de Marie d'Agoult. Ce philosophe, exilé politique en Belgique après 1851, avait rejoint Zurich au printemps 1856 pour enseigner la littérature française à l'École polytechnique ${ }^{23}$. Il avait trouvé un réconfort dans la fréquentation de Georg Herwegh et de son entourage d'artistes et d'écrivains, dont Wagner et probablement Semper. Par l'intermédiaire de Herwegh, Challemel-Lacour entre bientôt en relation avec Marie d'Agoult, dont il devient plus proche lorsqu'il regagne Paris après son amnistie en août 1859. Au sein de ses activités, la traduction de Semper se situe entre des travaux diffusant la pensée allemande (sa traduction de l'Histoire de la philosophie moderne de Heinrich Ritter en 1861 ou son Étude sur Guillaume de Humboldt en 1864) et des contributions de critique d'art dans la Revue moderne, par exemple sur les salons de 1864 et 1865. Intitulé « De l'ornementation et du style, de leur signification symbolique dans l'art», le texte est précédé d'une courte présentation de cet "architecte créateur» doublé d'un «théoricien profond» qui «achève la publication d'un grand ouvrage sur la Théorie $d u$ style $»^{24}$.

Lorsque paraît ce texte, l'historien et critique d'art Charles Blanc a déjà connaissance des travaux de Semper ${ }^{25}$. En septembre 1849, alors directeur de 1'administration des Beaux-arts, Blanc lui avait facilité l'accès aux collections assyriennes du Louvre. Wolfgang Herrmann rapporte qu'il s'était intéressé au projet d'une "Vergleichende Baulehre " "théorie comparative de la construction ») et avait laissé entrevoir à Semper une possible souscription du gouvernement pour l'achat de plusieurs centaines d'exemplaires, une fois l'ouvrage réalisé et traduit en français. Aucune de ces perspectives ne s'était concrétisée et leurs échanges ne semblent pas s'être poursuivis après le départ de Semper pour Londres. L'intérêt de Blanc se trouve ravivé lorsqu'il fonde la Gazette des beaux-arts en 1859. L'activité artistique à Dresde y est évoquée, dans les premières années, par plusieurs articles de Claire de Charnacé, signés

\footnotetext{
${ }^{22}$ Voir note 4.

${ }^{23}$ Eugène Grelé, L'odyssée d'un philosophe, aventures et tribulations de l'exil. Paul Challemel-Lacour. II. Le professeur, l'insurgé, le proscrit, Paris, E. Champion, 1920.

24 «Esthétique. Cours de M. Semper... », art. cit. (voir note 4), p. 513.

${ }^{25}$ Wolfgang Herrmann, op. cit. (voir note 2), p. 25.
} 
sous divers pseudonymes ${ }^{26}$. Ces textes n'évoquent pas l'architecte, néanmoins, dès 1859 paraît un curieux compte-rendu de «Leçons sur l'architecture, du professeur Semper $»^{27}$, ces conférences étant étrangement situées à Dresde, quand Semper enseigne à Zurich depuis 1855.

Si Charles Blanc n'avait que rapidement évoqué Semper, en 1863, à propos de la polychromie des temples grecs ${ }^{28}$, sa lecture de la traduction de Paul Challemel-Lacour est bien plus déterminante. Rappelons que dans le texte original, Semper entend mettre à jour des lois universelles qui régissent l'ornement, relevant à la fois de l'ordre naturel et de fondements anthropologiques. Il analyse les dispositifs formels de la parure corporelle, pour étendre progressivement l'étude aux ornements de l'architecture et des objets techniques. Blanc s'inspire de ce texte pour la première partie de sa Grammaire des arts décoratifs, publiée à p. 193 partir de $1870^{29}$. Après la Grammaire des arts $d u$ dessin consacrée aux principes du beau, cette seconde grammaire aborde ceux de l'embellissement selon des hypothèses comparables à celles de Semper, présupposant l'existence de forces vitales universelles qui orientent la création, qu'il s'agisse d'orner le corps ou l'édifice. La Grammaire des arts décoratifs devait initialement comporter trois parties, « du simple au composé », suivant « les trois aspects sous lesquels se présente le genre humain $»^{30}$, de l'individu, en passant par la famille, jusqu'à la société. La première partie, rassemblée en 1875 sous le titre L'art dans la parure et dans le vêtement ${ }^{31}$, examine l'ornement de la personne, la deuxième, Décoration intérieure de la maison $^{32}$, poursuit l'enquête à l'échelle domestique. Une troisième, restée inachevée ${ }^{33}$ au moment du décès de Blanc en 1882, devait aborder l'embellissement des sociétés, c'est-à-dire la décoration des villes et des monuments.

Dans cet ensemble qui synthétise nombre de lectures, l'un des chapitres sur la parure paraphrase très directement Semper : «Dans sa forme et dans ses ornements, le chapeau, comme le costume en général, doit se rapporter à ces trois forces de la nature et de la vie : l'attraction, la croissance et le mouvement $»^{34}$. Blanc rend hommage à Challemel-Lacour d'avoir rendu compréhensibles des idées qui lui étaient, en allemand, "inintelligibles » et ironise sur l'opacité de la version initiale :

\footnotetext{
${ }^{26}$ C. de Sault, «Christian Rauch », Gazette des beaux-arts (ci-après GBA), t. 4, oct. 1859, p. 5-17 ; Siegmund, « Correspondance de Dresde », GBA, t. 5, février 1860, p. 236-239 et t. 11, octobre 1861, pp. 373-374.

${ }^{27}$ Louis Meslin, « I. Illustrated Hand-book of Architecture, by James Fergusson, London. II. Vorlesungen über Architektur, von Professor Semper, Dresden, $1859 », G B A$, t. 3, juillet 1859, p. 117-123. L'identité de l'auteur de ce compte-rendu reste à préciser. Salomon Reinach cite également ces «Vorles. über Architektur» en bibliographie de son Manuel de philologie classique (Paris, Hachette, 1884, t. 2, appendice, p. 54).

${ }^{28}$ Charles Blanc, « Grammaire des arts du dessin », GBA, t. 14, mai 1863, p. 405 ; republié dans Grammaire des arts $d u$ dessin, Paris, J. Renouard, 1867.

${ }^{29}$ Du même, "Grammaire des arts décoratifs pour faire suite à la grammaire des arts du dessin. Introduction. Les lois générales de l'ornement » ( $1^{\mathrm{er}}$ article), GBA, t. 3, avril 1870, pp. 297-328.

${ }^{30} \mathrm{Du}$ même, «Grammaire des arts décoratifs. Division de l'ouvrage » (2 $2^{\text {er }}$ article), GBA, t. 3, juin 1870, (pp. 515-542), p. 516.

${ }^{31} \mathrm{Du}$ même, L'Art dans la parure et dans le vêtement, Paris, Renouard, 1875 (articles parus sous le titre «Grammaire des arts décoratifs » dans la $G B A$ entre juin 1870 et juillet 1874).

${ }^{32}$ Du même, Grammaire des arts décoratifs. Décoration de la maison, Paris, J. Renouard, 1881.

${ }^{33}$ Du même, «La Décoration des villes », Le Temps, n 6606, 23 mai 1879, n 6723, 17 sept. 1879, n 6734, 28 sept. $1879, \mathrm{n}^{\circ} 6760,24$ oct. $1879, \mathrm{n}^{\circ} 6767,31$ oct. 1879 (n.p.).

${ }^{34}$ Du même, « Grammaire des arts décoratifs. Chapitre VIII », GBA, t. 4, sept. 1870, (pp. 193-202), p. 193.
} 
Un des plus habiles architectes de l'Allemagne, M. Semper, qui est aussi un esprit original et spéculatif, un chercheur, a cru pouvoir ramener à trois principes l'ornementation de la figure humaine. Voici un exposé sommaire de ses idées, qui paraissent profondes dans leur obscurité, mais qui paraîtront plus profondes et plus belles, si nous parvenons, tout en les résumant, à les éclaircir ${ }^{35}$.

Blanc reprend les trois catégories de parure définies par Semper en référence aux dynamiques naturelles: les pendentifs, soumis à la pesanteur (boucles d'oreilles); les ornements annulaires qui rayonnent vers la périphérie (colliers, bracelets, couronne); les ornements de direction liés au mouvement vers l'avant (crinières, crêtes) (fig. 1). Si ces trois genres trouvent leur illustration dans des coiffures, Blanc suggère leur déplacement vers les édifices : « il en est des vêtements et des ornements appliqués à la figure humaine comme des créations de l'architecte $»^{36}$. Avant lui, Semper voyait dans les antéfixes l'équivalent des panaches des casques et dans les astragales l'analogue des ceintures. Plus que tout autre aspect, la quête de symboles universels, fondés sur les lois de la nature et susceptibles d'être saisis par tous, rassemble les projets esthétiques des deux théoriciens.

\section{Louis de Ronchaud : la tapisserie dans l'Antiquité}

La diffusion d'idées exprimées dans le premier volume de Der Stil s'effectue, au début des années 1870, par l'entremise d'un ami intime et confident de Marie d'Agoult, l'historien de l'art Louis de Ronchaud. Les affinités qui apparaissent déjà dans son Phidias ${ }^{37}$, publié en 1861, lorsqu'il s'intéresse aux revêtements des statues chryséléphantines et à la polychromie des temples grecs, reposent sur des références archéologiques communes, en particulier Karl Otfried Müller. De Ronchaud ne cite Semper qu'à partir de 1872, dans une suite d'articles sur l'usage des tapisseries dans l'Antiquité parus dans la Revue archéologique ${ }^{38}$. L'historien y réexamine l'idée d'un «illustre architecte allemand, M. Semper, auteur d'une Esthétique pratique, dont le premier volume est consacré à l'art textile », selon laquelle "l'art de tisser serait né avec l'art de bâtir $\aleph^{39}$. Des premières cloisons formées de végétaux entrelacés, en passant par les tissages colorés revêtant les parois de bois, jusqu'aux tapisseries associées aux murs de pierre, l'activité du tisserand précède la création architecturale :

L'importance toute particulière des draperies dans le système des constructions antiques [...] est telle que, jusque sous le régime de la pierre, la draperie conserve le privilège d'être la représentation légitime des idées de séparation et d'enceinte ${ }^{40}$.

\footnotetext{
35 Ibid., pp. 193-194.

${ }^{36}$ Ibid., p. 200.

${ }^{37}$ Louis de Ronchaud, Phidias, sa vie et ses ouvrages, Paris, Gide, 1861.

${ }^{38} \mathrm{Du}$ même, «Le Peplos d'Athéné. Étude sur les tapisseries dans l'Antiquité et sur leur emploi dans l'architecture et spécialement dans la décoration du Parthénon », Revue archéologique, vol. XXIII, avril 1872 , pp. 245-252 ; mai 1872, pp. 309-319, juin 1872, pp. 390-395 et vol. XXIV, août 1872, pp. 80-94.

${ }^{39}$ Ibid., p. 309.

${ }^{40}$ Ibid., pp. 309-310.
} 
Soulignant ainsi le rôle des étoffes dans la délimitation spatiale, de Ronchaud insiste également sur leur revalorisation dans l'analyse des systèmes monumentaux :

Quant au monument, il naquit, toujours selon M. Semper, du désir de fixer d'une manière durable un appareil de fête. Les décorations, les ornements, tapis, fleurs, festons, couronnes, qui avaient servi pour une solennité particulière, deviennent autant de motifs d'architecture. Dans le système du savant architecte de Hambourg, toutes les parties solides, bien que nécessaires pour soutiens, n'en sont pas moins d'ordre secondaire et faites pour être cachées. Le premier rôle, celui d'élément générateur, pour parler son langage, appartient aux tissus; la draperie est le principe qui domine l'architecture et qui préside à tous ses développements; chaque matière nouvelle employée aux tissus donne des motifs de forme et de couleur d'où naissent de perpétuelles modifications. L'enveloppement, le déguisement forment un caractère essentiel de la construction primitive, de l'art primitif ${ }^{41}$.

De Ronchaud juge cependant ce système « un peu absolu » et objecte que «l'art de tisser n'est peut-être pas aussi étroitement lié à l'art de bâtir». Il leur suppose plutôt deux évolutions parallèles; mais ne reconnaît pas moins à Semper d'avoir su "éclairer d'une lumière nouvelle l'histoire de l'art ancien, en appelant l'attention sur le rôle important de la draperie dans l'architecture $»^{42}$. L'auteur développe ensuite sa propre enquête, examinant l'usage des tentures dans l'habitation antique ainsi que la pratique des statues habillées. Il imagine enfin, d'après un passage d'Euripide, un système de décorations textiles formant une sorte de tente à l'intérieur du Parthénon, protégeant ainsi la statue de la déesse Athéna ${ }^{43}$.

Les théories textiles diffusées par de Ronchaud suivent l'hypothèse plus générale d'un transfert des motifs depuis les arts d'industrie vers l'architecture. Dans les années 1870 et 1880, cette idée a pris de l'importance dans les travaux d'historiens de l'art français, à partir p. 196 de sources également consultées par Semper. Les affinités sont particulièrement sensibles entre Der Stil et l'Histoire de l'art dans l'Antiquité de Georges Perrot et Charles Chipiez, qui mobilisent parfois la même iconographie (fig. 2). Chipiez avait lui-même, dans son Histoire critique des origines et de la formation des ordres grecs (1876), soutenu l'hypothèse d'une « influence des arts secondaires sur l'architecture monumentale $»^{44}$. Dans leur premier volume consacré à l'Égypte (1882), Perrot et Chipiez rendent hommage à l'architecte allemand :

Cet écrivain a montré le premier que le vannier, le tisserand et le potier, en travaillant les matières premières sur lesquelles s'exerce leur industrie, ont produit, par le seul jeu des procédés techniques, des combinaisons de lignes et de couleurs, des dessins dont l'ornemaniste s'est emparé dès qu'il a eu à décorer les murs, les corniches et les plafonds des édifices. Comme ces arts élémentaires étaient certainement plus anciens

\footnotetext{
${ }^{41}$ Ibid.

${ }^{4}$ Ibid., p. 311.

${ }^{43}$ Le passage d'Euripide est également commenté dans Der Stil in den technischen und tektonischen Künsten, oder Praktische Ästhetik, t. 1, Francfort / Main, Verlag für Kunst und Wissenschaft, 1860, p. 285.

${ }^{44}$ Charles Chipiez, Histoire critique des origines et de la formation des ordres grecs, Paris, ${ }^{\mathrm{ve}} \mathrm{A}$. Morel et $\mathrm{C}^{\mathrm{ie}}$, 1876, p. 355.
} 
que l'architecture, ces ornements n'ont certainement point passé des murailles sur les nattes, les étoffes et les pots : c'est le contraire qui est arrivé !45

Considérées d'abord par la critique et l'histoire de l'art, les idées de Semper sur les relations entre textile et architecture rencontrent plus tardivement le milieu architectural. Louis de Ronchaud, qui republie son interprétation en $1884^{46}$, présente ses théories sur la cella du Parthénon à la Société centrale des architectes le 21 mai $1885^{47}$, quelques mois après la parution d'un premier article sur Semper dans La Semaine des constructeurs ${ }^{48}$.

\section{Paris par Zurich, Londres et Vienne : Lawrence Harvey et Hans Semper}

C'est à un élève francophone de Semper, Lawrence Harvey, genevois d'origine anglaise, que l'on doit l'essentiel de la transmission de ses idées vers le milieu architectural français. Son résumé du premier tome de Der Stil dans la Revue générale de l'architecture en 1886-1887 ${ }^{49}$ est l'adaptation française d'une conférence qu'il avait donnée, en décembre 1884, au Royal Institute of British Architects (RIBA) de Londres. L'enseignement qu'il avait reçu à Zurich y est ravivé par une nouvelle lecture de Der Stil et par une enquête sur la période londonienne de Semper.

Fils d'un professeur de littérature anglaise installé à Genève, Lawrence Harvey, bien que non germanophone, avait entrepris des études d'architecture à l'Institut polytechnique de Zurich de 1864 à 1866 - en l'absence d'école supérieure à Genève et Lausanne. Ces études, suivies d'un passage à l'École des beaux-arts de Paris entre 1868 et 1871, sont engagées dans la perspective d'une carrière de maître d'œuvre à Londres, où il s'installe en novembre 1872. À la Bauschule de Zurich ${ }^{50}$, il avait suivi les cours d'histoire de l'architecture dispensés par Wilhelm Lübke et Gottfried Semper. Les perspectives intellectuelles ouvertes par ces leçons l'avaient enthousiasmé, malgré son incapacité initiale à saisir un enseignement dispensé en allemand. Ce sont précisément ces difficultés à comprendre la langue de Semper, de surcroît réputé mauvais orateur, qui le conduisent à acquérir Der Stil pour en approfondir l'étude. La culture littéraire familiale le rend notamment particulièrement sensible aux relations entre les origines respectives des formes artistiques et des langages.

\footnotetext{
${ }^{45}$ Georges Perrot et Charles Chipiez, Histoire de l'art dans l'antiquité, t. 1 Égypte, Paris, Hachette, 1882 , p. 807.

${ }^{46}$ Louis de Ronchaud, " De l'emploi de l'étoffe dans la division et la décoration des édifices de l'Antiquité », L'art, $10^{\mathrm{e}}$ année, 1884, t. II, pp. 74-76 et pp. 116-120; du même, La Tapisserie dans l'Antiquité. Le péplos d'Athené. La décoration du Parthénon restituée d'après un passage d'Euripide, Paris, Librairie de l'art J. Rouam, 1884.

${ }^{47}$ Du même, « La décoration intérieure de la cella », dans Au Parthénon, Paris, E. Leroux, 1886, pp. 26-86.

${ }^{48}$ « Semper et sa théorie de l'art», La Semaine des constructeurs, vol. 9, n ${ }^{\circ} 28,10$ janv. 1885, p. 329.

${ }^{49}$ Lawrence Harvey, «L'architecte Semper. Sa théorie sur l'origine des styles », RGATP, vol. XLIII, 1886, col. 58-63, 124-131 et 203-209 ; vol. XLIV, 1887, col. 18-23, 49-54, 97-104, 145-131, 193-202.

${ }^{50}$ Sur l'enseignement de Semper, voir Martin Fröhlich, Gottfried Semper als Entwerfer und Entwurfslehrer. Materialen zur Entwurfslehre im 19. Jahrhundert aus dem Zürcher Semper-Archiv, thèse, Zurich, 1974 ; Susanne Lüttmann, Gottfried Sempers «Vergleichende Baulehre ». Eine Quellenkritische Rekonstruktion, thèse, Zurich, 2008 et Martin Tschanz, die Bauschule am Eidgenössischen Polytechnikum in Zürich. Architekturlehre zur Zeit von Gottfried Semper (1855-1871), Zurich, gta Verlag, 2015.
} 
Son intérêt se ranime à Londres. Fin 1884, devenu membre du RIBA, Harvey inaugure une redécouverte des théories de Semper, alors relativement oublié ${ }^{51}$, dans une conférence et plusieurs publications ${ }^{52}$. Soucieux d'attirer l'attention, il met en avant sa qualité d'ancien élève et s'appuie, pour retracer sa trajectoire londonienne, sur des échanges avec Hans Semper $^{53}$. Harvey reformule les idées développées dans Der Stil dans une langue orale, p. 199 accessible et vivante, ponctuée d'anecdotes, avec une conscience aiguë de la nécessité d'une transposition simplifiée à destination d'un auditoire d'architectes. Il laisse entendre qu'Eugène Guillaume, ancien directeur de l'École des beaux-arts de Paris, lui aurait suggéré de « traduire l'œuvre de Semper en français au bénéfice des écoles d'art » ${ }^{54}$, mais que cette tâche était incompatible avec son activité d'architecte. L'entreprise de diffusion d'une théorie abrégée, telle qu'engagée à Londres, se prolonge néanmoins en direction de la presse architecturale parisienne, pour laquelle Harvey assure déjà, depuis 1882, des chroniques sur l'art de bâtir en Angleterre. Sa version des idées de Semper intervient dans le débat qu'avait suscité en octobre 1884 une conférence de l'architecte des magasins du Printemps, Paul Sédille, prônant une expression architecturale fondée sur le réalisme de la structure et sur la richesse polychrome des nouveaux matériaux fournis par l'industrie ${ }^{55}$. Dans La Semaine des constructeurs, un critique avait mis en doute les bénéfices d'une « construction apparente $»^{56}$ nécessitant de coûteux traitements, par rapport à l'emploi d'enduits et autres revêtements traditionnels qui assurent protection et expression esthétique. C'est dans ce contexte que paraît en janvier 1885 un compte rendu anonyme des interventions britanniques de Harvey sur «Semper et sa théorie de l'art », soulignant l'idée selon laquelle « il convient de recouvrir les matériaux par d'autres matériaux plus agréables à l'œil, ou autrement: que le vêtement philosophique prévaut aussi bien au point de vue de l'art qu'au point de vue des mœurs $\rangle^{57}$. Le mois suivant, Lawrence Harvey présente un «Exemple pratique de la théorie de Semper $»^{58}$ (fig. 3). Il s'agit de l'entrée d'un hôtel particulier qu'il a réalisé à Londres et dont «la conception architecturale et décorative sent, ainsi que Semper définit l'art, les flambeaux de

\footnotetext{
${ }^{51}$ À sa mort paraît une petite notice nécrologique: Thomas L. Donaldson, «The Late Gottfried Semper of Vienna », Royal Institute of British Architects. Transactions, 1878-1879, p. 233.

${ }^{52}$ Lawrence Harvey, "Gottfried Semper», The Architect, 18 oct. 1884, p. 243-244 ; du même, «Semper's Theory of Evolution in Architectural Ornament », conférence donnée le 15 déc. 1884, Transactions of the RIBA, 1885 , pp. 29-54 et pl. IX-XVII.

${ }^{53}$ Hans Semper lui aurait demandé d'enquêter à Londres sur les plans faits par son père pour le complexe de South-Kensington. Voir Lawrence Harvey, «l'Institut impérial britannique », La Construction moderne, vol. 8, 29 oct. 1892, (p. 38-42), p. 40. Voir aussi Harry F. Mallgrave, op. cit., p. 365 et p. 416, n. 35.

${ }^{54}$ The Architect, 20 déc. 1884, p. 407. Harvey écrit aussi à Hans Semper avoir été sollicité pour traduire Der Stil en anglais, proposition qu'il aurait déclinée avec les mêmes arguments (Harry F. Mallgrave, op. cit., p. 365).

${ }^{55}$ Paul Sédille, «L'architecture contemporaine et les industries d'art qui s'y rattachent», Revue des arts décoratifs, $5^{\mathrm{e}}$ année, 1884-1885, pp. 283-293.

${ }^{56}$ Émile Rivoalen, « Réflexions sur la conférence faite par Paul Sédille, architecte, à l'Union centrale des arts décoratifs le 22 octobre 1884 », RGATP, 1884, col.248-25 et, du même, « La construction apparente et 1'architecture de l'avenir », La Semaine des constructeurs, vol. 9, n² 21, 22 nov. 1884, pp. 244-246 et n 23,6 déc. 1884 , pp. $268-269$

${ }^{57}$ «Semper et sa théorie de l'art», art. cit. Ce compte rendu, dont l'auteur est probablement Paul Planat, est établi d'après les articles parus dans The Architect.

${ }^{58}$ Lawrence Harvey, "Exemple pratique de la théorie de Semper », La Semaine des constructeurs, vol. 9, $\mathrm{n}^{\circ} 34$, 21 fév. 1885 , pp. 402-404 et n 35, 28 fév. 1885, pp. 412-413.
} 
carnaval ; c'est un essai de poème en brique et en mortier $»^{59}$. Harvey insiste sur la définition de l'architecture comme art d'expression, «qui pare d'un masque réjouissant les réalités prosaïques $»^{60}$, tout en rapprochant cette conception de celle attribuée à l'architecte français Félix Duban, définissant l'architecture comme de la « décoration construite $»^{61}$.

Ces articles entraînent une vive polémique sur les rapports du beau et de l'utile et sur la définition d'une science esthétique appliquée à l'architecture, précédant la rupture entre le directeur de La Semaine des constructeurs - César Daly - et son rédacteur en chef - Paul Planat. La discussion sur Semper reprend, d'une part, dans la Revue générale de l'architecture, mensuel également dirigé par Daly, et d'autre part, dans le nouvel hebdomadaire fondé par Planat, La Construction moderne.

Dans la Revue générale, Lawrence Harvey est invité à présenter aux lecteurs français un long résumé de $\mathrm{Der} \mathrm{Stil}^{62}$. S'il insistait, auprès de ses confrères anglais, sur la période londonienne de Semper, cette contribution fait de ce dernier un homme «dont l'esprit a été vivifié par la pensée française ${ }^{63}$. L'effort d'adaptation opéré par Harvey pour résumer un texte si complexe dans une langue simple et vivante, à la portée du lectorat des architectes français, est tout aussi remarquable que dans son intervention au RIBA. Bien plus développé, l'article intitulé « L'architecte Semper. Sa théorie sur l'origine des styles » comprend deux chapitres. Le premier, « Notions générales d'esthétique. Influence des matières premières sur le style », présente la thèse centrale de Der Stil, qui «n'est autre chose que la démonstration des dettes que tous les arts ont contractées envers les petits métiers ${ }^{64}$, caractérisés en quatre ensembles : les arts textiles liés aux matières souples et résistantes, la céramique qui façonne des corps plastiques, la charpenterie qui assemble de longues pièces et enfin la taille de matériaux denses comme la pierre. Harvey insiste sur les interactions entre ces techniques : « les limites de chaque métier sont assez vagues $»^{65}$. Le développement sur l'art textile est ensuite privilégié. S'adressant aux architectes français, Harvey oppose implicitement les théories de Semper à celles des héritiers d'Eugène Viollet-le-Duc :

Semper maintient en effet que pendant toute l'Antiquité jusqu'à la fin du Moyen-Âge, la décoration a toujours été considérée comme un vêtement avec lequel on recouvrait la construction. C'est l'art gothique qui le premier a inauguré le principe que la construction toute nue était belle d'elle-même et qu'il fallait la rendre aussi évidente que possible, une manière de faire que Semper appelle porter ses côtes au dehors. Vêtir est la réalité même de l'art. C'est dit ! Le drapeau de Semper est déployé ! ${ }^{66}$

\footnotetext{
${ }^{59}$ Harvey avait précédemment publié ce bâtiment en Grande-Bretagne, en réponse à un détracteur anonyme (Lawrence Harvey, « Semper Theory of Evolution and its Practical Utility Illustrated », The Architect, 24 janv. 1885, p. 59).

${ }^{60}$ Ibid., p. 403.

${ }^{61}$ L'opposition entre les définitions de Labrouste (l'architecture comme construction ornée) et de Duban (décoration construite) avait été médiatisée par Charles Blanc, Les Artistes de mon temps, Paris, Firmin-Didot, 1876, p. 22.

${ }^{62}$ Lawrence Harvey, « L'architecte Semper. Sa théorie sur l'origine des styles », art. cit.

${ }^{63}$ Ibid., col. 58-59.

${ }^{64}$ Ibid., col. 63.

${ }^{65}$ Ibid., col. 130.

${ }^{66}$ Ibid., p. 131.
} 
Le second chapitre de cette étude résume le premier volume de Der Stil sous l'intitulé «Histoire du vêtement monumental» (fig. 4). Harvey y suit l'art textile depuis les «sauvages» jusqu'aux temps modernes, en passant par les Indes, la Chine, l'Assyrie, l'Égypte et la Grèce, au fil d'un récit où la construction «n'est qu'un squelette qui n'a d'autre but que de servir de support à un vêtement artistique ${ }^{67}$. Il insiste également sur les transferts des arts techniques vers l'architecture, montrant comment le mobilier assyrien permet d'expliquer la forme des chapiteaux de Persépolis, imitation en marbre de détails d'anciennes structures en bois revêtues de métal.

p. 202 Ces analyses qui assimilent la surface décorative des édifices à une enveloppe textile rencontrent les réflexions de César Daly. Dans l'article qui suit immédiatement celui de Harvey, il formule sa propre hypothèse d'une migration des motifs issus de techniques anciennes vers l'art de bâtir. Dissertant, à propos des tombeaux d'Asie mineure, sur l'influence des étoffes sur certaines constructions traditionnelles, il conclut sur la nécessité, pour «éclairer bien des points restés obscurs », d'étudier les «formes paléontologiques de l'architecture $\rangle^{68}$ (fig. 5).

La parution du compte rendu de Harvey coïncide avec un nouvel intérêt pour les réalisations de Semper dans la capitale autrichienne. En août 1884, un article de Paul Sédille sur l'architecture moderne à Vienne ${ }^{69}$ mentionnait déjà, mais de façon bien imprécise, ses écrits, en signalant une traduction inexistante de Der Stil. En 1886, Hans Semper, invité à présenter l'évolution architecturale en Allemagne et en Autriche dans La Construction moderne ${ }^{70}$, ouvre sa contribution en citant quelques extraits «de l'ouvrage sur Le Style » ${ }^{71}$. Paul Planat renchérit :

C'est là qu'il faut remonter si l'on veut savoir quels enseignements peut fournir l'Esthétique, produit du génie allemand ou anglais, appliquée à l'architecture ; si l'on veut se familiariser avec cette doctrine de l'évolution appliquée aux arts par Semper, et dont on a beaucoup parlé depuis, sans l'avoir toujours bien comprise; si l'on veut surtout y rechercher le premier et sérieux essai d'une histoire de l'art, exposée dans ses enchainements logiques ${ }^{72}$.

\footnotetext{
${ }^{67}$ Ibid., col. 19.

${ }^{68}$ César Daly, «Tombeaux lyciens et phrygiens taillés dans le roc », RGATP, vol. XLIV, (col. 104-109), col. 109. L'article est illustré par une planche de la tombe de Midas (pl. 31) empruntée à la Description de l'Asie Mineure de Charles Texier (1839), également commentée par Semper à partir de la même source.

${ }^{69}$ «Le professeur Semper, architecte de grand talent, a construit beaucoup en Allemagne. De plus, il a écrit plusieurs ouvrages intéressants, dont l'un, Le Style, a été traduit en français. » (Paul Sédille, «L'architecture moderne à Vienne », GBA, t. 30, $1^{\text {er }}$ déc. 1884, [pp. 481-491], p. 131).

${ }^{70}$ Paul Planat et Hans Semper, "L'architecture moderne en Allemagne et en Autriche », La Construction moderne, $\mathrm{n}^{\circ} 32,7$ août 1886 , pp. 519-520.

${ }^{71}$ Il s'agit d'un long passage sur l'emploi des trois ordres de l'architecture, de la Grèce antique à la Renaissance. (Der Stil in den technischen und tektonischen Künsten oder Praktische Ästhetik, t. 2, Munich, Bruckmann, 1863, pp. 476-477).

${ }^{2}$ Paul Planat et Hans Semper, art. cit, p. 519.
} 
Il insiste ainsi sur l'antériorité des travaux de Semper, répondant implicitement à César Daly au moment où celui-ci se pose en représentant d'une science esthétique appliquée à l'architecture ${ }^{73}$.

La transposition de Semper s'effectue parallèlement du côté des arts décoratifs lorsqu'Émile Reiber, dessinateur industriel d'origine alsacienne, publie, à partir de 1886, plusieurs traductions dans sa revue L'Art pour tous ${ }^{74}$. Militant actif pour une réforme de l'enseignement des arts techniques en France, Reiber actualise Wissenschaft, Industrie und Kunst [Science, industrie et art] au moment où se prépare à Paris l'Exposition universelle de 1889. Une notice biographique signée par Lawrence Harvey ${ }^{75}$ et quelques extraits commentés de Der Stil, notamment le passage consacré aux $"$ Écoles d'architecture du XIX ${ }^{\mathrm{e}}$ siècle $»^{76}$, accompagnent ensuite une version française de Die vier Elemente der Baukunst [Les Quatre éléments de l'architecture] $]^{77}$.

Lesquelles, parmi ces publications, sont à l'origine du passage que l'architecte Léon Labrouste consacre à Semper lorsqu'il rédige en 1892 son article $"$ Style » ${ }^{78}$ ? Semper n'est pas évoqué pour définir ce terme, mais son œuvre est citée, avec celle de John Ruskin ${ }^{79}$, au rang des philosophies artistiques contemporaines les plus pertinentes. Labrouste réinterprète librement Semper au prisme de ses propres convictions et il lui prête une définition de l'art de bâtir plutôt héritée de son père Henri Labrouste :

L'architecture ne saurait être fixe dans ses formes, sa destination résumant des besoins changeants, tandis que son principe, qui a pour base l'humanité, demeure toujours ; enfin, les matériaux doivent trouver dans la construction une application naturelle ${ }^{80}$.

p. 205 Il est frappant que Léon Labrouste classe 1'architecte allemand parmi les « idéalistes », contre les «matérialistes » représentés par Hippolyte Taine, au moment même où Alois Riegl critique la théorie de la détermination technique et matérielle prônée par les sempériens ${ }^{81}$. La libre adaptation de Labrouste s'est ensuite diffusée, l'article «Style » étant traduit dans une revue américaine ${ }^{82}$ et cité par Louis Cloquet, professeur d'architecture à l'université de Gand, dans son Traité d'architecture ${ }^{83}$.

\footnotetext{
${ }^{73}$ César Daly, « L'esthétique, la science, l'art et l'histoire, leurs apports », RGATP, vol. XLII, 1885, col. 64-75 et «Ce que c'est que l'esthétique », La Semaine des constructeurs, vol. IX, n 38, 21 mars 1885, p. 455.

${ }^{74}$ Voir note 5.

${ }^{75}$ Lawrence Harvey, «L'architecte Semper », L’Art pour tous, Bulletin, n 36, déc. 1888, n. p. et n 38 , fév. 1889 , n. p. Ces articles reprennent des éléments parus dans la RGATP, vol. XLIII, 1886, col. 58-63.

${ }^{76}$ Gottfried Semper, « De la situation des artistes et de l'industrie », L'Art pour tous, Bulletin, ${ }^{\circ}$ 37, janv. 1889, n.p. et «Les écoles d'architecture du XIX ${ }^{\mathrm{e}}$ siècle », L'Art pour tous, Bulletin, $\mathrm{n}^{\circ} 39$ et 40 , mars et avril 1889 (traductions, par Émile Reiber, d'extraits des Prolégomènes de Der Stil).

${ }^{77}$ Voir note 5.

${ }^{78}$ Léon Labrouste, «Style », in : Paul Planat (éd.), Encyclopédie de l'architecture et de la construction moderne, Paris, Dujardin et $C^{\text {ie }}$, vol. 6, 1892, pp. 543-566.

${ }^{79}$ Lawrence Harvey avait rédigé un long compte rendu des Sept lampes de l'architecture dans La Construction moderne en 1890.

${ }^{80}$ Ibid., p. 548.

${ }^{81}$ Alois Riegl, Stilfragen : Grundlegung zur einer Geschichte der Ornamentik, Berlin, G. Siemens, 1893, p. VI.

${ }^{82}$ Léon Labrouste, «Style », The American Architect and Building News, vol. XLIV, 5 mai 1894, p. 47.

${ }^{83}$ Louis Cloquet, "Théories actuelles de l'architecture», in: Traité d'architecture, t. 5, Paris/Liège, Ch. Béranger, 1901, pp. 113-114. La réception de Gottfried Semper en Belgique mériterait d'être approfondie. Charles Buls livre dès octobre 1869 un compte rendu de Der Stil dans la Revue de Belgique, puis rédige en 1876-
} 
Les déplacements de l'iconographie de Der Stil vers des ouvrages en français mériteraient également d'être mieux étudiés, puisque les phénomènes de translation et d'adaptation concernent aussi les illustrations. Certaines figures, qui proviennent de sources documentaires largement partagées dans les cercles archéologiques européens, se retrouvent dans de nombreuses autres publications. Quant aux dessins spécifiquement réalisés par Semper pour Der Stil, hormis dans les articles de Harvey, ils ne sont pas reproduits dans les publications que nous avons repérées. Certaines images ressurgissent toutefois au fil d'itinéraires inattendus. La gravure de la «Restitution d'un temple étrusque d'après Semper» retrouvée dans l'Histoire des styles d'architecture ${ }^{84}$ (fig. 6) de l'architecte Étienne Barberot ne provient pas directement de la planche couleur du premier volume de Der Stil, mais de la traduction française de l'Essai d'histoire de l'art (1886) de Wilhelm Lübke ${ }^{85}$ qui transcrivait déjà la restauration de Semper, initialement d'après sa première publication dans le Deutsches Kunstblatt en $1855^{86}$.

Ainsi, bien que peu de textes soient intégralement traduits, de multiples versions françaises des travaux de Semper circulent dans les deux dernières décennies du siècle. Selon Harvey, Semper affirmait ne pas écrire pour les 4 lecteurs paresseux $\|^{87}$, mais pour ceux capables d'examiner de façon critique ses arguments. L'architecte anglais suggère-t-il ainsi que Der Stil était précisément conçu pour engendrer des réécritures actives ? Orientées par la diversité de ses champs d'intérêt - critique artistique, archéologie, architecture -, ces versions suivent également, dans l'espace européen, des trajectoires complexes entre Zurich, Londres, Vienne et Paris. Semper y est évoqué, de façon récurrente, comme un représentant de l'esthétique germanique, souvent pour dénoncer l'opacité de sa langue et de ses concepts, mais aussi parfois pour souligner l'originalité d'une approche non surplombante, placée du côté de la pratique et proche des objets. Le philosophe Victor Basch, en 1912, est néanmoins le premier à positionner véritablement Semper dans une histoire de l'esthétique allemande contemporaine $^{88}$. En faisant de l'architecte l'initiateur, avant Ernst Grosse, Wilhelm Wundt et August Schmarsow, d'une "science de l'art» attentive aux caractéristiques techniques des œuvres ainsi qu'à leur dimension sociologique et anthropologique, il inaugure une nouvelle lecture de Der Stil.

1877 un ensemble d'articles sur les arts techniques. Voir Marcel Smets, Charles Buls : Les principes de l'art urbain, Liège, Mardaga, 1995, pp. 37-54.

${ }^{84}$ Étienne Barberot, Histoire des styles d'architecture dans tous les pays, depuis les temps anciens jusqu'à nos jours, Paris, Baudry, 1891, t. 1, fig. 156, p. 145.

${ }^{85}$ Wilhelm Lübke, Essai d'histoire de l'art, trad. Charles Adolphe Koëlla, Paris/Stuttgart, J. Rouam/Ebner \& Seubert, 1886, t. 1, fig. 189, p. 191.

${ }^{86}$ Du même, Geschichte der Architektur von den ältesten Zeiten bis auf die Gegenwart dargestellt, t. 1, Leipzig, E. A. Seemann, 1884 , p. 256 : la restitution du temple étrusque est ici évoquée à partir de sa publication par Semper dans le Deutsches Kunstblatt, ${ }^{\circ} 9,1^{\text {er }}$ mars 1855, p. 80.

${ }^{87}$ Lawrence Harvey, « Semper's Theory of Evolution in Architectural Ornament », art. cit., p. 54.

${ }^{88}$ Victor Basch, «De l'esthétique allemande contemporaine», Revue philosophique de la France et de l'Étranger, t. LXXIII, janvier-juin 1912, (pp. 167-190), pp. 175-181. 


\section{Illustrations}

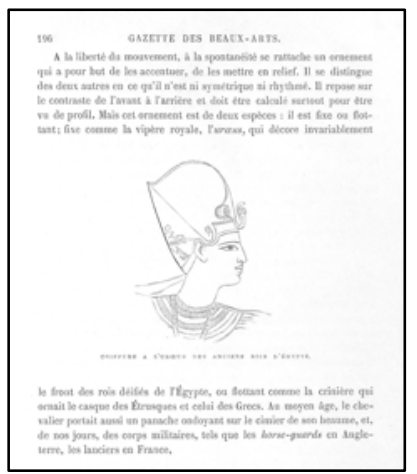

Fig. 1. « Coiffure à l'Urœus des anciens rois d’Égypte », Charles Blanc, " Grammaire des arts décoratifs », Gazette des beaux-arts, t. 4, 1870, p. 196.

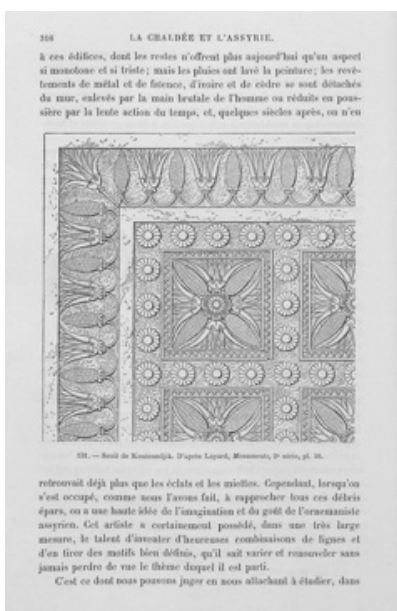

Fig. 2. "Seuil de Kouioundjik », dans Georges Perrot et Charles Chipiez, Histoire de l'art dans l'Antiquité, t. 2, 1884, p. 316, d'après Austen Henry Layard, A Second Series of the Monuments of Nineveh (1853).

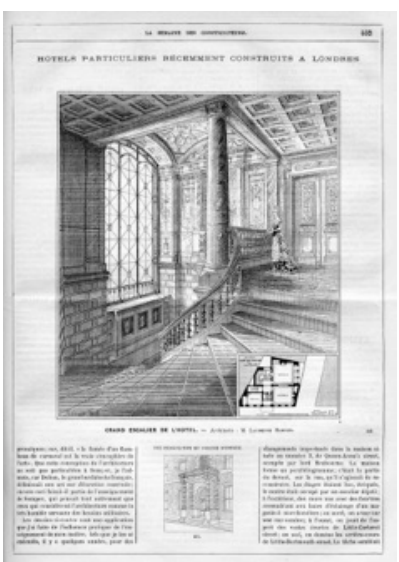

Fig. 3. Lawrence Harvey, « Exemple pratique de la théorie de Semper », La semaine des constructeurs, vol. 9,1885 , p. 403.

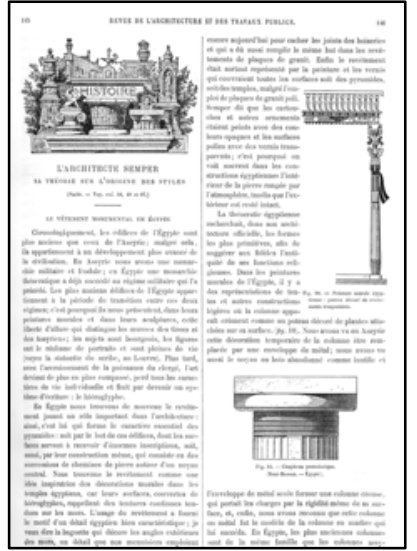

Fig. 4. Lawrence Harvey, «L'architecte Semper. Sa théorie sur l'origine des styles ", Revue générale de l'architecture, vol. XLIV, 1887, col. 145-146.

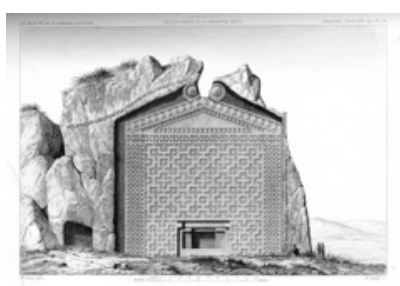

Fig. 5. «Tombeau du Roi Midas Midas », Revue générale de l'architecture, vol. XLIV, 1887, pl. 31 , d'après Charles Texier, Description de l'Asie mineure (1839), t. 1, pl. 56.

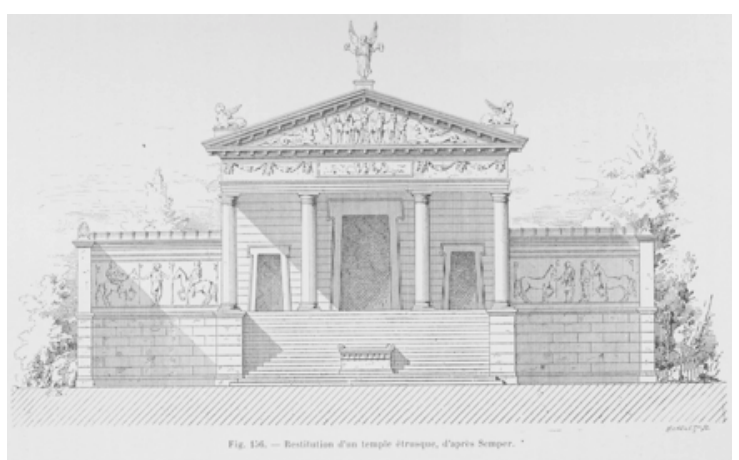

Fig. 6. « Restitution d'un temple étrusque d'après Semper », dans Étienne Barberot, Histoire des styles d'architecture..., 1891, t. 1, fig. 156, d'après Wilhelm Lübke, Essai sur l'histoire de l'art (1886), p. 191. 